\title{
Symmetries of Spin Calogero Models ${ }^{\star}$
}

\author{
Vincent CAUDRELIER ${ }^{\dagger}$ and Nicolas CRAMPÉ $\ddagger$ \\ $\dagger$ Centre for Mathematical Science, City University, \\ Northampton Square, London, EC1V OHB, United Kingdom \\ E-mail:v.caudrelier@city.ac.uk \\ ¥ International School for Advanced Studies, Via Beirut 2-4, 34014 Trieste, Italy \\ E-mail: crampe@sissa.it
}

Received September 24, 2008, in final form December 17, 2008; Published online December 23, 2008

Original article is available at http://www.emis.de/journals/SIGMA/2008/090/

\begin{abstract}
We investigate the symmetry algebras of integrable spin Calogero systems constructed from Dunkl operators associated to finite Coxeter groups. Based on two explicit examples, we show that the common view of associating one symmetry algebra to a given Coxeter group $W$ is wrong. More precisely, the symmetry algebra heavily depends on the representation of $W$ on the spins. We prove this by identifying two different symmetry algebras for a $B_{L}$ spin Calogero model and three for $G_{2}$ spin Calogero model. They are all related to the half-loop algebra and its twisted versions. Some of the result are extended to any finite Coxeter group.
\end{abstract}

Key words: Calogero models; symmetry algebra; twisted half-loop algebra

2000 Mathematics Subject Classification: 70H06; 81R12; 81R50

\section{Introduction}

Dunkl operators [1] were introduced by C.F. Dunkl as part of a program on polynomials in several variables with reflection symmetries related to finite reflection groups (or equivalently finite Coxeter groups). Besides this important mathematical motivation, they quickly became fundamental objects in the study of integrable quantum mechanical many-body systems introduced by F. Calogero and B. Sutherland [2,3] and generalized to any Weyl group in [4]. Indeed they can be viewed as generalizations of momentum operators with which they share the crucial property of forming an Abelian algebra. This fact allows one to implement the exchange operator formalism described in [5] producing many-body integrable Hamiltonians. In [6, 7], it was realized that this could be pushed further by representing the Coxeter group on spins ${ }^{1}$. This gave $L$-particle integrable spin Calogero systems related to the group $A_{L}$. Then one could play several games such as changing the type of potential and/or the underlying group while maintaining integrability.

Another big step was taken in [8] where the "FRT formalism" [9], so powerful with spin chain type integrable models, was used in connection with the algebraic properties of the Dunkl operators to construct the symmetry algebra. By this, we mean the algebra of the operators commuting with the Hamiltonian of the system (and its hierarchy). In the case of [8], it is a non Abelian infinite dimensional algebra which contains the whole hierarchy of the integrable $A_{L}$ spin Sutherland model in its centre: the Yangian of $\mathfrak{g l}_{N}$ [10]. This type of symmetry algebra is a very efficient tool to study and, in particular, to compute explicitly the spectrum, the eigenvectors and the correlation functions of a large class of integrable models: Sutherland model [11, 12,

${ }^{\star}$ This paper is a contribution to the Special Issue on Dunkl Operators and Related Topics. The full collection is available at http://www.emis.de/journals/SIGMA/Dunkl_operators.html

${ }^{1}$ Here the spin is to be understood as an internal degree of freedom taking $N$ different values. 
13], Haldane-Shastry spin model [14, 15, 16, 17], non-linear Schrödinger equation [18, 19] and Hubbard model [20].

The method developed in [8] was then derived in various ways, again accommodating various groups and potentials, to identify the corresponding symmetry algebras. From there, following several results in the literature, some standard views imposed themselves as granted: for instance, the symmetry algebra of an $A_{L}$ Sutherland type model [8] should be a Yangian while the Calogero counterpart (without harmonic confinement) should be the corresponding half-loop algebra. Similarly, for $B_{L}$ Sutherland models, the reflection algebra [21] should be symmetry algebra [22] with the corresponding twisted half-loop algebra as symmetry algebra of the Calogero counterpart (this paper).

Actually, we show in this paper that this is not true and in general, it is not enough to specify the type of potential and the underlying Coxeter group to identify the correct symmetry algebra of the associated integrable model. In addition, one has to give the spin representation of the Coxeter group. This is examplified in this paper by working with rational Dunkl operators (hence producing Calogero type potentials) based on two particular finite Coxeter groups: $B_{L}$ and $G_{2}$. In the first three sections, we recall the background on finite reflection groups, half-loop algebras and the link to dynamical spin integrable systems that is necessary for our purposes. In Section 5, we deal with $B_{L}$ spin Calogero model for which we identify two different symmetry algebras by choosing two different ways of representing $B_{L}$ on the spin configuration space. In Section 6 , we apply the same strategy with three different spin representations of $G_{2}$ to obtain three different symmetry algebras. All the symmetry algebras are related to the $\mathfrak{g l}_{N}$ half-loop algebra and its twisted versions. Various generalizations of our discussion are collected in Section 7. Our conclusions are gathered in the last section.

\section{Dunkl operators associated to finite reflection groups}

Here we recall the ingredients we need from the theory of finite reflection groups (see e.g. [23]) and Dunkl operators [1]. A reflection in the real Euclidean space $\mathbb{R}^{L}$ endowed with the scalar product $\left(e_{i}, e_{j}\right)=\delta_{i j}$, where $\left\{e_{i}\right\}_{i=1, \ldots, L}$ are the canonical basis vectors of $\mathbb{R}^{L}$, is a linear operator $s_{\alpha}\left(\alpha \in \mathbb{R}^{L}, \alpha \neq 0\right)$ on $\mathbb{R}^{L}$ defined by

$$
\forall \mu \in \mathbb{R}^{L}, \quad s_{\alpha}(\mu)=\mu-2 \frac{(\mu, \alpha)}{(\alpha, \alpha)} \alpha .
$$

It sends the vector $\alpha$ to $-\alpha$ and leaves invariant the hyperplane $H_{\alpha}$ orthogonal to $\alpha$. A root system $\Phi$ is a subset of $\mathbb{R}^{L}$ satisfying, for all $\alpha \in \Phi$,

$$
\begin{aligned}
& (\lambda \in \mathbb{R}, \alpha \in \Phi \quad \text { and } \quad \lambda \alpha \in \Phi) \Rightarrow \lambda= \pm 1, \\
& s_{\alpha} \Phi=\Phi .
\end{aligned}
$$

$\Phi$ is a finite set and the group generated by $\left\{s_{\alpha} \mid \alpha \in \Phi\right\}$ is a finite subgroup $W$ of $O\left(\mathbb{R}^{L}\right)$ called the reflection group associated to $\Phi$. Any root system can be written as $\Phi=\Phi^{+} \cup\left(-\Phi^{+}\right)\left(\Phi^{+}\right.$ is called the positive root system). A simple system $\Delta$ is a subset of $\Phi$ such that $\Delta$ is a vector space basis of $\operatorname{span}_{\mathbb{R}} \Phi$ and each $\alpha \in \Phi$ is a linear combination of $\Delta$ with coefficients all of the same sign.

An important result is that the reflection group $W$ is generated by $\left\{s_{\alpha} \mid \alpha \in \Delta\right\}$ subject only to the relations, for $\alpha, \beta \in \Delta$

$$
\left(s_{\alpha} s_{\beta}\right)^{m(\alpha, \beta)}=1
$$

with $m(\alpha, \alpha)=1$ and $m(\alpha, \beta)>1(\alpha \neq \beta)$. That is to say, by definition, $W$ is a Coxeter group. The possible values of $m(\alpha, \beta)$ such that the group $W$ is finite provides a classification of the finite reflection groups. 
To each Coxeter group $W$, one can associate Dunkl operators which are differential operators acting on functions $\varphi: \mathbb{R}^{L} \rightarrow \mathbb{C}$. Let us define the natural action of $W$ on such functions by

$$
(\hat{s} \varphi)(\mu)=\varphi\left(s^{-1}(\mu)\right)
$$

with $s \in W$ and $\mu \in \mathbb{R}^{L}$. The map $s \mapsto \hat{s}$ is a representation of the group $W$. A function $k: \Phi \rightarrow \mathbb{C}$ is called a multiplicity function if

$$
\forall \alpha, \beta \in \Phi, \quad k(\beta)=k\left(s_{\alpha}(\beta)\right) .
$$

Let $k$ be given. For $\xi \in \mathbb{R}^{L}$, the Dunkl operator $d_{\xi}$ acts on $C^{1}\left(\mathbb{R}^{L}\right)$ and is defined by

$$
d_{\xi}=-i \partial_{\xi}+i \sum_{\alpha \in \Phi^{+}} k(\alpha) \frac{(\alpha, \xi)}{(\alpha, x)} \hat{s}_{\alpha}
$$

where $\partial_{\xi}$ is the derivative in the direction $\xi, x=\left(x_{1}, \ldots, x_{L}\right)^{t}$ with $x_{i}$ the operator multiplication by $x_{i}$. Note that our choice of Dunkl operators yields hermitian operators provided $k$ is real-valued. This is important for quantum mechanical applications. The Dunkl operators do not depend on the choice of the positive root system $\Phi^{+}$and have the following fundamental properties $^{2}$

Proposition 1 ([1]). The Dunkl operators are $W$-equivariant, i.e. for any $s \in W$, we get

$$
\hat{s} d_{\xi} \hat{s}^{-1}=d_{s(\xi)} \text {. }
$$

The Dunkl operators commute, i.e. for any $\xi, \zeta \in \mathbb{R}^{L}$, we get

$$
d_{\xi} d_{\zeta}=d_{\zeta} d_{\xi}
$$

\section{Half-loop algebra and twists}

This section is meant to give necessary definitions and notations to handle algebraic structures related to some integrable models.

The $\mathfrak{g l}_{N}$ half-loop algebra ${ }^{3}$ is the unital algebra $\mathfrak{g l}_{N}[z]$ of polynomials in an indeterminate $z$ with coefficients in $\mathfrak{g l}_{N}$ : it is generated by $\left\{e_{i j} z^{n} \mid 1 \leq i, j \leq N, n=0,1,2, \ldots\right\}$ where $e_{i j}$ are the generators of $\mathfrak{g l}_{N}$ satisfying the commutation relations

$$
\left[e_{i j}, e_{k \ell}\right]=\delta_{j k} e_{i \ell}-\delta_{i \ell} e_{k j} .
$$

Let $E_{i j}$ be the matrix with 1 on the entry $(i, j)$ and 0 elsewhere. The map $e_{i j} \mapsto E_{i j}$ provides a representation of $\mathfrak{g l}_{N}$. In the following, we will use auxiliary space notations to simplify computations. We define the monodromy matrix of the half-loop algebra as follows

$$
T(u)=\sum_{i, j=1}^{N} E_{i j} \otimes \sum_{n=0}^{\infty} \frac{e_{j i} z^{n}}{u^{n+1}}=\sum_{i, j=1}^{N} \frac{E_{i j} \otimes e_{j i}}{u-z} \in \operatorname{End}\left(\mathbb{C}^{N}\right) \otimes \mathfrak{g l}_{N}[z]\left[\left[u^{-1}\right]\right],
$$

where $u$ is a formal parameter called the spectral parameter and $\operatorname{End}\left(\mathbb{C}^{N}\right)$ is the auxiliary space used to pack nicely the generators. The defining relations of the half-loop algebra can be written

$$
\left[T_{a}(u), T_{b}(v)\right]=\left[T_{a}(u)+T_{b}(v), r_{a b}(u-v)\right],
$$

\footnotetext{
${ }^{2}$ Strictly speaking, the proof in [1] is given for slightly different Dunkl operators simply related to ours by the gauge transformation $d_{\xi} \mapsto \phi^{-1}(x) d_{\xi} \phi(x)$ where $\phi$ is a $W$-invariant function $\phi(s(x))=\phi(x)$ for all $s \in W$.

${ }^{3}$ Also sometimes called Gaudin algebra.
} 
where $a$ and $b$ denote two copies of the auxiliary space $\operatorname{End}\left(\mathbb{C}^{N}\right), T_{a}(u)=T(u) \otimes \mathbb{I}_{N}, T_{b}(u)=$ $\mathbb{I}_{N} \otimes T(u), \mathbb{I}_{N}$ is the identity in $\operatorname{End}\left(\mathbb{C}^{N}\right)$, and

$$
r_{a b}(u)=\sum_{i, j=1}^{N} \frac{E_{i j} \otimes E_{j i}}{u} \equiv \frac{P_{a b}}{u}
$$

is a classical $r$-matrix solution of the classical Yang-Baxter equation [24].

Let $\sigma \in \operatorname{End}\left(\mathbb{C}^{N}\right)$ with $\sigma^{n}=\mathbb{I}_{N}$ for some $n \in \mathbb{N}$. The eigenvalues of $\sigma$ are the powers of the $n$-th root of unity $\tau=e^{\frac{2 i \pi}{n}}$. We denote $A$ the inner automorphism of $\mathfrak{g l}_{N}$ associated to $\sigma$. Its action on the generators of $\mathfrak{g l}_{N}$ is given by

$$
A: \quad e_{i j} \mapsto(\sigma)_{j n} e_{m n}\left(\sigma^{-1}\right)_{m i} .
$$

We extend this action to $\mathfrak{g l}_{N}[z]$ in the natural way and we define

$$
\mathfrak{g l}_{N}[z]^{\sigma}=\left\{M(z) \in \mathfrak{g l}_{N}[z] \mid A M(z)=M(\tau z)\right\} .
$$

One can see that $\mathfrak{g l}_{N}[z]^{\sigma}$ is a subalgebra of $\mathfrak{g l}_{N}[z]$ which is called the (inner) twisted half-loop algebra of $\operatorname{order} n$. Now we proceed to derive the commutation relations of this algebra. For $k \in \mathbb{N}$, we introduce the projectors

$$
\mathcal{P}_{k}=\frac{1}{n} \sum_{j=0}^{n-1} \tau^{-j k} A^{j}
$$

Note that there are only $n$ such projectors as $\mathcal{P}_{n+k}=\mathcal{P}_{k}$. This allows us to define a surjective projection map from $\mathfrak{g l}_{N}[z]$ to $\mathfrak{g l}_{N}[z]^{\sigma}$ by $e_{i j} z^{k} \mapsto \mathcal{P}_{k} e_{i j} z^{k}$. In turn, this maps $T(u)$ to

$$
B(u)=\frac{1}{n} \sum_{j=0}^{n-1} \tau^{j} \sigma^{j} T\left(\tau^{j} u\right) \sigma^{-j} \in \operatorname{End}\left(\mathbb{C}^{N}\right) \otimes \mathfrak{g l}_{N}[z]^{\sigma}\left[\left[u^{-1}\right]\right],
$$

which contains the generators of $\mathfrak{g l}_{N}[z]^{\sigma}$ by construction. Next we have [25]

Proposition 2. $B(u)$ satisfies the symmetry property

$$
\forall j=0, \ldots, n-1, \quad B(u)=\tau^{j} \sigma^{j} B\left(\tau^{j} u\right) \sigma^{-j},
$$

and encodes the commutation relations of $\mathfrak{g l}_{N}[z]^{\sigma}$ as

$$
\left[B_{a}(u), B_{b}(v)\right]=\frac{1}{n} \sum_{j=0}^{n-1}\left[\tau^{j} B_{a}(u)+B_{b}(v), \frac{\left(\sigma^{j}\right)_{b} P_{a b}\left(\sigma^{-j}\right)_{b}}{u-\tau^{j} v}\right] .
$$

For our purposes, the following facts are important. If $b(u)=\operatorname{Tr} B(u)$, where the trace is taken over the auxiliary space $\operatorname{End}\left(\mathbb{C}^{N}\right)$, then it follows from $(3.3)$ that

$$
[b(u), B(v)]=0, \quad[b(u), b(v)]=0 .
$$

\section{Link with integrable dynamical spin models}

The Dunkl operators allow us to construct integrable Hamiltonians by implementing the strategy discussed in [5]. Indeed, any polynomial in the Dunkl operators commutes with the independent and two by two commuting operators $\left\{d_{e_{i}} \mid i=1, \ldots, L\right\}$. When it is of order 2 , this polynomial can usually be regarded as an Hamiltonian which is then integrable. 
The next step is to consider Hamiltonians for particles with $N$ internal degrees of freedom. The construction to introduce these "spins" has been pioneered in $[6,7]$ and the symmetry of the corresponding models has been shown in [8]. The point is that the Dunkl operators only act on the positions $x_{i}$ while now the wavefunctions become $\varphi(x \mid s), x \in \mathbb{R}^{L}, s \in\left(\mathbb{C}^{N}\right)^{\otimes M}$. Let us remark that $M$ may be different from $L$ in general. So one has to come up with a method allowing the Dunkl operators to act on the spins while maintaining their nice properties. This is done with a suitable projector

$$
\Lambda=\frac{1}{|W|} \sum_{w \in W} \hat{w} R_{w}
$$

Note that we require that the wavefunctions satisfy the following generalized statistics ${ }^{4}$ for $w \in W$,

$$
\hat{w} R_{w} \varphi(x \mid s)=\varphi(x \mid s),
$$

where $R: W \rightarrow \operatorname{End}\left(\left(\mathbb{C}^{N}\right)^{\otimes M}\right)$ is a representation of $W$.

One then acts with $d_{\xi} \Lambda$ on the wavefunctions. Unfortunately, usually $\left[d_{\xi} \Lambda, d_{\zeta} \Lambda\right] \neq 0$ so the idea to use directly polynomials in $d_{\xi} \Lambda$ to get an integrable hierarchy fails. The idea of [8] is to use the monodromy matrix formalism and the underlying algebraic structures to circumvent this problem. In our context, consider a set of $\mathrm{M}$ vectors $\left\{\xi_{1}, \ldots, \xi_{M}\right\}$. Then, the matrix

$$
T_{a}(u)=\sum_{k=1}^{M} \frac{P_{a k}}{u-d_{\xi_{k}}}
$$

provides a representation of the half-loop algebra (3.1). At this point, let us emphasize that the auxiliary space has no 'physical' meaning (in the sense it does not act on the wavefunctions) but it is a powerful and convenient tool to deal with the infinite algebras we are interested in. Then, the 'physical' operators are contained in the entries of the matrix.

One obtains $B(u)$ through (3.2) with the properties (3.4). The crucial part is to show that, for a suitable choice of $\xi$ and of the twist, $B(u) \Lambda$ is also a representation of the algebra (3.3). To prove this statement, it is sufficient to show that $\Lambda B(u) \Lambda=B(u) \Lambda$ which is guaranteed by, for $g$ any generator of $W$,

$$
\hat{g} R_{g} B(u) \Lambda=B(u) \Lambda .
$$

Finally, using properties (3.4) for $B(u) \Lambda$, we conclude that $\operatorname{Tr} B(u) \Lambda$ provides a hierarchy of commuting operators when expanding in powers of $u^{-1}$. To prove the integrability, it is now sufficient to prove that in this hierarchy there are $L$ independent quantities. Noting that these operators are polynomials in $d_{\xi}$ times $\Lambda$, we may choose one of them as the Hamiltonian acting both on the position and spin degrees of freedom of the wavefunctions. As a by-product, but not the least, we prove that this Hamiltonian has the twisted half-loop algebra for symmetry algebra.

It is very important to realize that it is the choice of $R$, the representation of $W$ on the spins, together with the requirement (4.1), that imposes the form of $B(u)$ and hence the symmetry algebra. This is the essential message of this paper.

In the rest of this paper, we detail this construction for two Coxeter groups, $B_{L}$ and $G_{2}$. In particular, we get the surprising result that spin Calogero-type integrable models built from Dunkl operators associated to the same Coxeter group can have different symmetry algebras.

\footnotetext{
${ }^{4}$ Called this way because in the case $W=A_{L}$ and with the permutation representation on positions and spins, these conditions amount to consider bosonic wavefunctions.
} 


\section{$5 \quad$ Reflection group $B_{L}$}

We consider the Coxeter group $B_{L}$. It is generated by $L$ elements $t_{1}, \ldots, t_{L-1}$ (generating the Coxeter group $A_{L-1}$ ) and $r$ with defining relations

$$
\begin{aligned}
& r^{2}=1, \quad t_{i}^{2}=1, \quad i=1, \ldots, L-1, \\
& \left(t_{i} t_{i+1}\right)^{3}=1, \quad i=1, \ldots, L-2, \quad\left(t_{i} t_{j}\right)^{2}=1, \quad|i-j|>1, \\
& \left(r t_{L-1}\right)^{4}=1, \quad\left(r t_{j}\right)^{2}=1, \quad j \neq L-1 .
\end{aligned}
$$

\subsection{The usual $B_{L}$ spin Calogero model}

To fix ideas, we start with a known model [26]. We choose the usual positive root system $\Phi^{+}=\left\{e_{i} \pm e_{j}, e_{k} \mid 1 \leq i<j \leq L, 1 \leq k \leq L\right\}$. Then, the representation of the generators of $B_{L}$ associated to simple roots are

$$
t_{i}=s_{e_{i}-e_{i+1}}=\left(\begin{array}{cccc}
\mathbb{I}_{i-1} & & & \\
& 0 & 1 & \\
& 1 & 0 & \\
& & & \mathbb{I}_{L-i-1}
\end{array}\right) \quad \text { and } \quad r=s_{e_{L}}=\left(\begin{array}{ll}
\mathbb{I}_{L-1} & \\
& -1
\end{array}\right) \text {, }
$$

where $\mathbb{I}_{j}$ is the $j \times j$ unit matrix. In this case, the Dunkl operators are given by

$$
d_{e_{k}} \equiv d_{k}=-i \partial_{e_{k}}+i k_{l} \sum_{j \neq k}\left(\frac{1}{x_{k}-x_{j}} \hat{s}_{e_{k}-e_{j}}+\frac{1}{x_{k}+x_{j}} \hat{s}_{e_{k}+e_{j}}\right)+\frac{i k_{s}}{x_{k}} \hat{s}_{e_{k}} .
$$

The constants $k_{l}$ and $k_{s}$ are the two arbitrary values that the multiplicity function can take on $B_{L}$. The action of $B_{L}$ on $\left(\mathbb{C}^{N}\right)^{\otimes L}$ is taken as

$$
R_{t_{i}}=P_{i i+1} \quad \text { and } \quad R_{r}=Q_{L},
$$

where $Q \in \operatorname{End}\left(\mathbb{C}^{N}\right)$ satisfies $Q^{2}=\mathbb{I}_{N}$. We apply (3.2) to

$$
T_{a}(u)=\sum_{k=1}^{L} \frac{P_{a k}}{u-d_{k}}
$$

with $\sigma=Q$ (so $\tau=-1$ and $n=2$ ). We get

$$
B_{a}(u)=\frac{1}{2}\left(T_{a}(u)-Q_{a} T_{a}(-u) Q_{a}\right)=\frac{1}{2} \sum_{k=1}^{L}\left(\frac{P_{a k}}{u-d_{k}}+\frac{Q_{a} P_{a k} Q_{a}}{u+d_{k}}\right) .
$$

To prove that $B(u) \Lambda$ satisfies (3.3), it is enough to prove

$$
\hat{r} R_{r} B(u)=B(u) \hat{r} R_{r} \quad \text { and } \quad \hat{t}_{i} R_{t_{i}} B(u)=B(u) \hat{t}_{i} R_{t_{i}} .
$$

This is readily seen as

$$
\hat{r} R_{r} B(u)=\frac{1}{2} \sum_{k=1}^{L-1}\left(\frac{P_{a k}}{u-d_{k}}+\frac{Q_{a} P_{a k} Q_{a}}{u+d_{k}}\right) \hat{r} R_{r}+\frac{1}{2}\left(\frac{Q_{L} P_{a L} Q_{L}}{u-\hat{r} d_{L} \hat{r}}+\frac{Q_{L} Q_{a} P_{a L} Q_{a} Q_{L}}{u+\hat{r} d_{L} \hat{r}}\right) \hat{r} R_{r}
$$

and $\hat{r} d_{L} \hat{r}=d_{r e_{L}}=d_{-e_{L}}=-d_{L}$ (recall also that $Q^{2}=\mathbb{I}_{N}$ ). Similarly, one looks at the $i$-th and $i+1$-th term in the second relation in (5.2) and uses $\hat{t}_{i} d_{i} \hat{t}_{i}=d_{t_{i} e_{i}}=d_{e_{i+1}}=d_{i+1}$ together with $P_{i i+1} P_{a i} P_{i i+1}=P_{a i+1}$. 
One can now extract the commuting quantities by expanding $b(u) \Lambda$ in powers of $u^{-1}$. One gets

$$
b(u) \Lambda=\sum_{n=0}^{\infty} \frac{1+(-1)^{n}}{2 u^{n+1}} \sum_{k=1}^{L} d_{k}^{n} \Lambda .
$$

We see that only $J_{2 n}=\sum_{k=1}^{L} d_{k}^{2 n} \Lambda$ yield non trivial quantities. In particular, one gets the usual $B_{L}$ spin Calogero Hamiltonian

$$
H=J_{2}=-\sum_{j=1}^{L} \partial_{e_{j}}^{2}+k_{l} \sum_{m \neq j}\left(\frac{k_{l}-P_{m j}}{\left(x_{m}-x_{j}\right)^{2}}+\frac{k_{l}-Q_{j} P_{m j} Q_{j}}{\left(x_{m}+x_{j}\right)^{2}}\right)+k_{s} \sum_{j=1}^{L} \frac{k_{s}-Q_{j}}{x_{j}^{2}} .
$$

Then, we get the well-known result [26] that this hamiltonian is integrable by proving that $J_{2}, J_{4}, \ldots, J_{2 L}$ are independent. In addition, as explained in Section 4, we prove that this model has for symmetry the twisted half-loop algebra of order 2 . This result may be also obtained by considering the symmetry of the $B_{L}$ Sutherland model proved in [22] and taking the suitable limit as explained in [8] for the $A_{L}$ case.

\section{$5.2 \quad$ Another $B_{L}$ spin Calogero model}

Here we follow the approach of [27] and use another representation of $B_{L}$ for the spins while keeping the same on $\mathbb{R}^{L}$. Let us fix $\mu \in \mathbb{R}^{L}$. The orbit of the vector $\mu$ under the group $B_{L}$ is written

$$
\left\{w(\mu) \mid w \in B_{L}\right\} \equiv\left\{\mu_{1}, \ldots, \mu_{M}\right\}
$$

for some $M \in \mathbb{N}$ and with $\mu_{j} \in \mathbb{R}^{L}$. The group $B_{L}$ acts transitively on this set, i.e. for any $\mu_{j}, \mu_{k}$ there exists $w \in B_{L}$ such that $w\left(\mu_{j}\right)=\mu_{k}$. For any $w \in B_{L}$, we obtain an action on the set $\{1, \ldots, M\}$ by defining

$$
\check{w}(i)=j \quad \text { iff } \quad w\left(\mu_{i}\right)=\mu_{j} .
$$

The map $w \mapsto \check{w}$ is a representation of $B_{L}$. We can now define a representation of $B_{L}$ on $\left(\mathbb{C}^{N}\right)^{\otimes M}$ as the map $w \mapsto R_{w}$ where

$$
R_{w} v_{1} \otimes \cdots \otimes v_{M}=v_{\breve{w}^{-1}(1)} \otimes \cdots \otimes v_{\breve{w}^{-1}(M)}
$$

with $v_{i} \in \mathbb{C}^{N}$.

At this stage, one can see that

$$
T_{a}(u)=\sum_{k=1}^{M} \frac{P_{a k}}{u-d_{\mu_{k}}}
$$

commutes with the projector $\Lambda$ in the chosen representation. Indeed, for each $w \in B_{L}$

$$
\hat{w} R_{w} \sum_{k=1}^{M} \frac{P_{a k}}{u-d_{\mu_{k}}}=\sum_{k=1}^{M} \frac{P_{a \check{w}(k)}}{u-d_{w\left(\mu_{k}\right)}} \hat{w} R_{w}=\sum_{n=1}^{M} \frac{P_{a n}}{u-d_{\mu_{n}}} \hat{w} R_{w},
$$

where the last equality is obtained by relabelling the sum according to (5.5). Thus, $T(u) \Lambda$ satisfies the half-loop algebra relations and $\operatorname{Tr} T(u) \Lambda \equiv t(u) \Lambda$ provides the commuting elements. 
So we find that all the $B_{L}$ spin Calogero Hamiltonians obtained in this way (e.g. in [27]) have the half-loop algebra as symmetry algebra.

As an example, we apply this to $\mu=e_{1}$, the first vector of the canonical basis of $\mathbb{R}^{L}$. Then the orbit contains $2 L$ elements $\pm e_{j}, j=1, \ldots, L$ which we order as follows $\left(e_{1},-e_{1}, \ldots, e_{L},-e_{L}\right) \equiv$ $\left(\mu_{1}, \mu_{\overline{1}}, \ldots, \mu_{L}, \mu_{\bar{L}}\right)$. Then, by inspection we get

$$
\check{r}(j)=\left\{\begin{array}{ll}
\bar{L}, & j=L, \\
L, & j=\bar{L}, \\
j, & \text { otherwise }
\end{array} \quad \text { and } \quad \check{t}_{i}(j)= \begin{cases}i+1, & j=i, \\
i, & j=i+1, \\
\overline{i+1}, & j=\bar{i} \\
\bar{i}, & j=\overline{i+1} \\
j, & \text { otherwise. }\end{cases}\right.
$$

This gives

$$
R_{r}=P_{L, \bar{L}}, \quad R_{t_{i}}=P_{i, i+1} P_{\bar{i}, \overline{i+1}} .
$$

We get

$$
T_{a}(u)=\sum_{k=1}^{L} \frac{P_{a k}}{u-d_{\mu_{k}}}+\frac{P_{a \bar{k}}}{u-d_{\mu_{\bar{k}}}}=\sum_{k=1}^{L} \frac{P_{a k}}{u-d_{k}}+\frac{P_{a \bar{k}}}{u+d_{k}},
$$

where the Dunkl operators are given by (5.1). The following $B_{L}$ spin Calogero Hamiltonian has the half-loop algebra as symmetry algebra

$$
H=-\sum_{j=1}^{L} \partial_{e_{j}}^{2}+k_{l} \sum_{m \neq j}\left(\frac{k_{l}-P_{m j} P_{\bar{m} \bar{j}}}{\left(x_{m}-x_{j}\right)^{2}}+\frac{k_{l}-P_{m \bar{j}} P_{\bar{m} j}}{\left(x_{m}+x_{j}\right)^{2}}\right)+k_{s} \sum_{j=1}^{L} \frac{k_{s}-P_{j \bar{j}}}{x_{j}^{2}}
$$

As previously, $\operatorname{Tr} T(u) \Lambda$ provides only the even conserved quantities $J_{2 k}=\sum_{j=1}^{L} d_{j}^{2 k} \Lambda$ but it is sufficient to prove the integrability since $J_{2}, J_{4}, \ldots, J_{2 L}$ are again independent. Comparing (5.4) and (5.7), it is manifest that the only difference lies in the action on the spins, the potentials being indentical. Yet, these two systems based on $B_{L}$ have different symmetry algebras. It is also interesting to remark that (5.7) represents a system of $L$ particles on the line with two spin degrees of freedom attached to each particle.

\section{Group $G_{2}$}

We consider the Coxeter group $G_{2}$ (which is the dihedral group $I_{2}(6)$ of order 12 ). It is generated by 2 elements $t$ and $r$ subject only to the following relations

$$
r^{2}=1, \quad t^{2}=1, \quad(t r)^{6}=1 .
$$

It may also be generated by 2 elements $a$ and $b$ subject only to the following relations

$$
a^{6}=1, \quad b^{2}=1, \quad b a=a^{-1} b .
$$

The isomorphism between the two presentations reads

$$
a \mapsto t r \quad \text { and } \quad b \mapsto t .
$$




\subsection{Model with three particles and six spins}

The choice in this section for the positive roots of $I_{2}(6)$ in $\mathbb{R}^{3}$ are $\Phi^{+}=\left\{e_{1}-e_{2}, e_{3}-e_{1}, e_{3}-e_{2}\right\} \cup$ $\left\{-2 e_{1}+e_{2}+e_{3}, e_{1}-2 e_{2}+e_{3},-e_{1}-e_{2}+2 e_{3}\right\}$. Then, the the representation of the generators of $I_{2}(6)$ associated to simple roots are

$$
t=s_{e_{1}-e_{2}}=\left(\begin{array}{ccc}
0 & 1 & 0 \\
1 & 0 & 0 \\
0 & 0 & 1
\end{array}\right) \quad \text { and } \quad r=s_{-2 e_{1}+e_{2}+e_{3}}=\frac{1}{3}\left(\begin{array}{ccc}
-1 & 2 & 2 \\
2 & 2 & -1 \\
2 & -1 & 2
\end{array}\right) .
$$

To write down the Dunkl operators explicitly, it is useful to express all the reflections in terms of $r$ and $t$

$$
s_{e_{1}-2 e_{2}+e_{3}}=\text { trt }, \quad s_{e_{1}-e_{3}}=r t r, \quad s_{e_{2}-e_{3}}=\text { trtrt }, \quad s_{e_{1}+e_{2}-2 e_{3}}=\text { rtrtr } .
$$

We give only one example as the other ones are computed similarly:

$$
\begin{aligned}
d_{e_{1}}= & -i \partial_{e_{1}}+i k_{s}\left(\frac{1}{x_{1}-x_{2}} \hat{t}+\frac{1}{x_{1}-x_{3}} \widehat{r t r}\right) \\
& +i k_{l}\left(\frac{1}{x_{1}-2 x_{2}+x_{3}} \widehat{t r t}-2 \frac{1}{-2 x_{1}+x_{2}+x_{3}} \hat{r}+\frac{1}{x_{1}+x_{2}-2 x_{3}} \widehat{r t r t r}\right) .
\end{aligned}
$$

The action on the spins is defined as in Section 5.2 replacing the group $B_{L}$ by $I_{2}(6)$. That is, we define for $\mu \in \mathbb{R}^{3}$,

$$
\left\{\mu_{1}, \ldots, \mu_{M}\right\}=\left\{w(\mu) \mid w \in I_{2}(6)\right\}
$$

which provides a representation $R_{w}$ acting on spins. Thus, we have again that

$$
T_{a}(u)=\sum_{k=1}^{M} \frac{P_{a k}}{u-d_{\mu_{k}}}
$$

commutes with the projector $\Lambda$ in the chosen representation (the proof follows the same lines than the one in Section 5.2). We conclude that the half-loop algebra is the symmetry algebra of the integrable hierarchy contained in $\operatorname{Tr} T(u) \Lambda$.

We give an example. We may choose $\mu=e_{1}$. Then the orbit is

$$
\begin{gathered}
\left\{\mu_{1}=e_{1}, \mu_{2}=e_{2}, \mu_{3}=e_{3}, \mu_{\overline{1}}=\frac{1}{3}\left(-e_{1}+2 e_{2}+2 e_{3}\right),\right. \\
\left.\mu_{\overline{2}}=\frac{1}{3}\left(2 e_{1}-e_{2}+2 e_{3}\right), \mu_{\overline{3}}=\frac{1}{3}\left(2 e_{1}+2 e_{2}-e_{3}\right)\right\} .
\end{gathered}
$$

So, we get $M=6$. We deduce the following representation of $I_{2}(6)$ on $\left(\mathbb{C}^{N}\right)^{\otimes 6}$

$$
R_{t}=P_{12} P_{\overline{1} \overline{2}} \quad \text { and } \quad R_{r}=P_{1 \overline{1}} P_{2 \overline{3}} P_{3 \overline{2}} .
$$

The coefficient in front of $u^{-2}$ in the expansion of $t(u) \Lambda$ is proportional to the total momentum $P=-i \partial_{1}-i \partial_{2}-i \partial_{3}$ and the one in front of $u^{-3}$ is proportional to the Hamiltonian and reads

$$
H=-\sum_{i=1}^{3} \partial_{e_{i}}^{2}+k_{s} \sum_{m \neq j} \frac{k_{s}-P_{m j} P_{\bar{m} \bar{j}}}{\left(x_{m}-x_{j}\right)^{2}}+k_{l} \sum_{n \neq m \neq j} \frac{k_{l}-P_{n \bar{n}} P_{j \bar{m}} P_{m \bar{j}}}{\left(-2 x_{n}+x_{m}+x_{j}\right)^{2}} .
$$

By direct computation, we can show that the coefficients in front of $u^{-4}, u^{-5}$ and $u^{-6}$ are not independent of $P$ and $H$ whereas $J_{6}$, the one in front of $u^{-7}$, is independent of them. Then $P, H$ and $J_{6}$ provides 3 independent conserved quantites which proves the integrability of $H$. Let us remark that the degree in $d_{i}$ of the conserved quantities (i.e. 1, 2,6) are in agreement with the degrees of the invariant polynomials by the Coxeter group $I_{2}(6)$. In fact, it is not a coincidence since the conserved quantities we constructed may be seen as polynomials in terms of the variables $d_{i}$ and are, by construction, invariant under $I_{2}(6)$. 


\subsection{Model with three particles and three spins}

We keep the same positive roots as in Section 6.1. Then, the action of $I_{2}(6)$ on $\mathbb{R}^{3}$ is still the one defined by (6.1) but we take now the following representation of $I_{2}(6)$ on $\left(\mathbb{C}^{N}\right)^{\otimes 3}$

$$
R_{r}=P_{23} Q_{1} Q_{2} Q_{3}, \quad R_{t}=P_{12},
$$

where $Q^{2}=\mathbb{I}_{N}$. The projector may be written as follows

$$
\begin{aligned}
& \Lambda=\Lambda_{Q} \Lambda_{P}=\Lambda_{P} \Lambda_{Q}, \\
& \Lambda_{Q}=\frac{1}{2}\left(1+(\widehat{t r})^{3} Q_{1} Q_{2} Q_{3}\right), \\
& \Lambda_{P}=\frac{1}{6}\left(1+\widehat{t} P_{12}+\widehat{t r t r t} P_{23}+\widehat{r t r t} P_{12} P_{23}+\widehat{t r t r} P_{23} P_{12}+\widehat{r t r} P_{13}\right) .
\end{aligned}
$$

The projector $\Lambda_{P}$ is the usual symmetriser of the eigenfunctions for bosons and $\Lambda_{Q}$ yields

$$
\phi\left(x_{1}, x_{2}, x_{3} \mid s_{1}, s_{2}, s_{3}\right)=\phi\left(-x_{1}+2 X,-x_{2}+2 X,-x_{3}+2 X \mid s_{1}^{*}, s_{2}^{*}, s_{3}^{*}\right),
$$

where $s^{*}=Q s$ and $X=\frac{1}{3}\left(x_{1}+x_{2}+x_{3}\right)$ is the center of mass of the three particles.

The monodromy matrix is taken to be (with $d_{i} \equiv d_{e_{i}}$ ),

$$
T_{a}(u)=\frac{P_{a 1}}{u-d_{1}}+\frac{P_{a 2}}{u-d_{2}}+\frac{P_{a 3}}{u-d_{3}} .
$$

We apply a slight variant of (3.2) and define

$$
\begin{aligned}
\hat{B}_{a}(u) & =T_{a}(u)-Q_{a} T_{a}(-u+D) Q_{a} \\
& =\frac{P_{a 1}}{u-d_{1}}+\frac{P_{a 2}}{u-d_{2}}+\frac{P_{a 3}}{u-d_{3}}+\frac{Q_{1} P_{a 1} Q_{1}}{u+d_{1}-D}+\frac{Q_{2} P_{a 2} Q_{2}}{u+d_{2}-D}+\frac{Q_{3} P_{a 3} Q_{3}}{u+d_{3}-D},
\end{aligned}
$$

where $D=\frac{2}{3}\left(d_{1}+d_{2}+d_{3}\right)$. $\hat{B}(u)$ satisfies the following commutation relations

$$
\left[\hat{B}_{a}(u), \hat{B}_{b}(v)\right]=\frac{1}{2}\left[\hat{B}_{a}(u)+\hat{B}_{b}(v), \frac{P_{a b}}{u-v}\right]+\frac{1}{2}\left[-\hat{B}_{a}(u)+\hat{B}_{b}(v), \frac{Q_{a} P_{a b} Q_{a}}{u+v-D}\right] .
$$

This algebra is isomorphic to the twisted half-loop algebra of order $2: \hat{B}(u) \mapsto B(u+D / 2)$. The crucial points now are that $\hat{B}(u)$ commutes with $\Lambda$ and we still have $[\operatorname{Tr} \hat{B}(u), \operatorname{Tr} \hat{B}(v)]=0$. Then, the coefficient of $u^{-2}$ in $b(u) \Lambda$ is proportional to the total momentum $P=-i \partial_{1}-i \partial_{2}-i \partial_{3}$ and the one of $u^{-3}$ contains the following Hamiltonian, studied in [28],

$$
H=d_{1}^{2}+d_{2}^{2}+d_{3}^{2}=-\sum_{i=1}^{3} \partial_{e_{i}}^{2}+k_{s} \sum_{m \neq j} \frac{k_{s}-P_{m j}}{\left(x_{m}-x_{j}\right)^{2}}+k_{l} \sum_{n \neq m \neq j} \frac{k_{l}-P_{m j} Q_{n} Q_{m} Q_{j}}{\left(-2 x_{n}+x_{m}+x_{j}\right)^{2}} .
$$

As before, the coefficients of $u^{-4}, u^{-5}$ and $u^{-6}$ are not independent of $P$ and $H$. It is again $J_{6}$, the coefficient of $u^{-7}$, which provides the third independent conserved quantity and allows us to prove that the previous Hamiltonian is integrable.

Concerning the symmetry algebra, one has to be careful since the commutation relations (6.2) depend on the operator $D$ which is proportional to the total momentum. We can talk about the symmetry of the model (6.3) only in the sectors where the total momentum $P$ has a given value $p$. This is not a problem as the states are defined by three quantum numbers, $p$ being one of them and in the sectors of fixed $p$, the symmetry algebra is isomorphic to the twisted half-loop algebra. For example, in the center of the mass frame $(p=0)$, the symmetry algebra of (6.3) is the usual twisted half-loop algebra of order 2 . 


\subsection{Model with two particles and two spins}

Our choice for the simple roots of $I_{2}(6)$ in $\mathbb{R}^{2}$ is $e_{1}-e_{2}$ and $-e_{1}+\cot \left(\frac{\pi}{12}\right) e_{2}$. Then the other 4 positive roots are

$$
e_{1}-\tan \left(\frac{\pi}{12}\right) e_{2}, \quad e_{1}+\tan \left(\frac{\pi}{12}\right) e_{2}, \quad e_{1}+e_{2}, \quad e_{1}+\cot \left(\frac{\pi}{12}\right) e_{2} .
$$

Then, the action of both generators of $I_{2}(6)$ on $\mathbb{R}^{2}$ is given explicitly by

$$
b=\left(\begin{array}{ll}
0 & 1 \\
1 & 0
\end{array}\right) \quad \text { and } \quad a=\left(\begin{array}{cc}
\cos \left(\frac{\pi}{3}\right) & -\sin \left(\frac{\pi}{3}\right) \\
\sin \left(\frac{\pi}{3}\right) & \cos \left(\frac{\pi}{3}\right)
\end{array}\right) .
$$

It is useful to express all the reflections in terms of $a$ and $b$

$$
\begin{aligned}
& s_{e_{1}-e_{2}}=b, \quad s_{e_{1}-\tan \left(\frac{\pi}{12}\right) e_{2}}=a b, \quad s_{e_{1}+\tan \left(\frac{\pi}{12}\right) e_{2}}=a^{2} b, \\
& s_{e_{1}+e_{2}}=b a^{3}=a^{3} b, \quad s_{e_{1}+\cot \left(\frac{\pi}{12}\right) e_{2}}=b a^{2}, \quad s_{-e_{1}+\cot \left(\frac{\pi}{12}\right) e_{2}}=b a .
\end{aligned}
$$

Then, for example, we get

$$
\begin{aligned}
d_{e_{1}}= & -i \partial_{e_{1}}+i k_{s}\left(\frac{1}{x_{1}-x_{2}} \hat{b}+\frac{1}{x_{1}+(2-\sqrt{3}) x_{2}} \hat{a}^{2} \hat{b}+\frac{1}{x_{1}+(2+\sqrt{3}) x_{2}} \hat{b} \hat{a}^{2}\right) \\
& +i k_{l}\left(\frac{1}{x_{1}-(2-\sqrt{3}) x_{2}} \hat{a} \hat{b}+\frac{1}{x_{1}+x_{2}} \hat{a}^{3} b+\frac{1}{x_{1}-(2+\sqrt{3}) x_{2}} \hat{b} \hat{a}\right),
\end{aligned}
$$

where we have used $\tan \left(\frac{\pi}{12}\right)=2-\sqrt{3}$ and $\cot \left(\frac{\pi}{12}\right)=2+\sqrt{3}$. We define the action on $\left(\mathbb{C}^{N}\right)^{\otimes 2}$ by

$$
R_{a}=Q_{1} Q_{2}^{-1}, \quad R_{b}=P_{12}
$$

where $Q^{6}=\mathbb{I}_{N}$. The projector $\Lambda$ may be written as follows

$$
\begin{aligned}
& \Lambda=\Lambda_{Q} \Lambda_{P}=\Lambda_{P} \Lambda_{Q}, \\
& \Lambda_{P}=\frac{1}{2}\left(1+\hat{b} P_{12}\right), \\
& \Lambda_{Q}=\frac{1}{6}\left(1+\hat{a} Q_{1} Q_{2}^{-1}+\hat{a}^{2}\left(Q_{1} Q_{2}^{-1}\right)^{2}+\hat{a}^{3}\left(Q_{2} Q_{1}^{-1}\right)^{3}+\hat{a}^{4}\left(Q_{1} Q_{2}^{-1}\right)^{4}+\hat{a}^{5}\left(Q_{2} Q_{1}^{-1}\right)^{5}\right) .
\end{aligned}
$$

So, it is easy to see that the eigenfunctions are totally symmetric $\phi\left(x_{1}, x_{2} \mid s_{1}, s_{2}\right)=\phi\left(x_{2}, x_{1} \mid s_{2}, s_{1}\right)$ and satisfy the additional relation

$$
\phi\left(x_{1}, x_{2} \mid s_{1}, s_{2}\right)=\phi\left(\frac{x_{1}+\sqrt{3} x_{2}}{2}, \frac{x_{2}-\sqrt{3} x_{2}}{2} \mid Q s_{1}, Q^{-1} s_{2}\right) .
$$

It turns out to be convenient to map $\mathbb{R}^{2}$ into $\mathbb{C}$. Let us define

$$
d=d_{e_{1}}+i d_{e_{2}} \quad \text { and } \quad \bar{d}=d_{e_{1}}-i d_{e_{2}}
$$

and similarly for $\partial, \bar{\partial}$. We write the Dunkl operators in terms of $z=x_{1}+i x_{2}$ and $\bar{z}=x_{1}-i x_{2}$

$$
d=-i \partial+2 \sum_{j=0}^{2}\left(\frac{i k_{s}}{i \tau^{2 j} z+\bar{z}} \hat{b} \hat{a}^{2 j}+\frac{i k_{l}}{i \tau^{2 j+1} z+\bar{z}} \hat{b} \hat{a}^{2 j+1}\right),
$$




$$
\bar{d}=-i \bar{\partial}+2 \sum_{j=0}^{2}\left(\frac{i k_{s}}{z-i \tau^{2 j} \bar{z}} \hat{a}^{2 j} \hat{b}+\frac{i k_{l}}{z-i \tau^{2 j+1} \bar{z}} \hat{a}^{2 j+1} \hat{b}\right),
$$

where $\tau=\exp (i \pi / 3)$. The action of the generators of $I_{2}(6)$ on these Dunkl operators are

$$
\hat{a} d=\tau^{-1} d \hat{a}, \quad \hat{a} \bar{d}=\tau \bar{d} \hat{a}, \quad \hat{b} d=i \bar{d} \hat{b} \quad \text { and } \quad \hat{b} \bar{d}=-i d \hat{b} .
$$

Now, we introduce

$$
T_{a}(u)=\frac{P_{a 1}}{u-d}+\frac{P_{a 2}}{u-i \bar{d}}
$$

and also

$$
\hat{B}_{a}(u)=\sum_{j=0}^{5} \tau^{j} Q_{a}^{-j} T_{a}\left((u-d \bar{d}) \tau^{j}\right) Q_{a}^{j}=\sum_{j=0}^{5} \frac{Q_{a}^{-j} P_{a 1} Q_{a}^{j}}{u-d \bar{d}-\tau^{-j} d}+\frac{Q_{a}^{-j} P_{a 2} Q_{a}^{j}}{u-d \bar{d}-i \tau^{-j} \bar{d}} .
$$

This satisfies the commutation relations of the twisted half-loop algebra of order 6 with a shift of $d \bar{d}$ in the spectral parameters. We can show by direct investigation that $\hat{B}(u)$ commutes with the projector defined by (6.4). As usual, the series $\operatorname{Tr} \hat{B}(u) \Lambda$ provides the conserved quantities. We can show that 2 are independent: the coefficients in front of $u^{-2}$ and $u^{-7}$ proportional respectively to

$$
H=d \bar{d} \quad \text { and } \quad J_{6}=d^{6}-\bar{d}^{6}+2(d \bar{d})^{6} .
$$

Explicitly, the Hamiltonian is

$$
H=-\partial_{z} \partial_{\bar{z}}+4 i \sum_{j=0}^{2}\left(k_{s} \tau^{2 j} \frac{Q_{1}^{-2 j} P_{12} Q_{1}^{2 j}-k_{s}}{\left(z-i \tau^{2 j} \bar{z}\right)^{2}}+k_{l} \tau^{2 j+1} \frac{Q_{1}^{-(2 j+1)} P_{12} Q_{1}^{2 j+1}-k_{l}}{\left(z-i \tau^{2 j+1} \bar{z}\right)^{2}}\right) .
$$

As in Section 6.2, the commutation relations satisfied by $\hat{B}(u)$ depend on one operator of our theory $(H)$. Thus, in each sector of fixed energy, the symmetry algebra of the integrable hierarchy is isomorphic to the twisted half-loop algebra of order 6 .

\section{Generalizations}

Let us remark that the construction of Section 6.3 for $I_{2}(6)$ extends to $I_{2}(m)$, a presentation of which is

$$
a^{m}=1, \quad b^{2}=1, \quad b a=a^{-1} b .
$$

The results follow by taking $Q$ with $Q^{m}=\mathbb{I}_{N}$ and $\tau=e^{\frac{2 i \pi}{m}}$. In the expressions for the Dunkl, one has to modify the sum and the two independent operators are to be found at orders $u^{3}$ and $u^{m+1}$. The relevant symmetry algebra is the twisted half-loop algebra of order $m$.

Another general result holds: the construction of the spin representation described in Sections 5.2 and 6.1 applies to any finite Coxeter group $W$ as explained in [27]. So fixing $\mu$ and denoting $\left\{\mu_{1}, \ldots, \mu_{M}\right\}$ the orbit of $\mu$ under $W$, the representation $R$ as defined in (5.6) is always well-defined on $\left(\mathbb{C}^{N}\right)^{\otimes M}$ and the monodromy matrix

$$
T_{a}(u)=\sum_{k=1}^{M} \frac{P_{a k}}{u-d_{\mu_{k}}}
$$


commutes with the projector $\Lambda$ in the chosen representation. So we find that all the $W$ spin Calogero Hamiltonians obtained in this way have the half-loop algebra as symmetry algebra.

At this stage, it is worth mentioning that potentially physically interesting integrable Hamiltonians can always be constructed. Indeed, from the general theory of invariant polynomials under finite Coxeter groups, the polynomial of order two in the variables is always invariant. This means that Hamiltonians of the form

$$
-\sum_{j=1}^{L} \partial_{e_{i}}^{2}+\text { interactions terms }
$$

can always be extracted from the hierarchy. We note however that $M$ can be different from $L$ so these systems may represent particles with more than one spin attached to them.

\section{Conclusions}

We have shown that, as far as symmetry is concerned, speaking of a $W$ spin Calogero model is not enough. Even if the root structure of $W$ provides the form of the interaction term in Hamiltonians constructed from Dunkl operators based on $W$, the symmetry algebra heavily depends on the choice of representation of $W$ on the spins. Using the method pioneered in [8], we showed this explicitly for the case of the Coxeter groups $B_{L}$ and $I_{2}(6)$. For the former, two different symmetry algebras were constructed while we proposed three of them for the latter. We stress that in all cases, the identification of the symmetry algebra is an original result in itself.

It should be noted that one systematic method, based on [27], always yields the same symmetry algebra (here the half-loop algebra for Calogero type models) for all Coxeter groups. However, there seems to be no systematic understanding of how to produce other possibilities such as those of Section 5.1 for $B_{L}$ and Sections 6.2, 6.3 for $G_{2}$. In physical terms, such an understanding would allow one to control the number of spins $M$ and make it coincide (if desired) with the number of particles $L$. Indeed, it all amounts to finding a construction of spin representations based on orbits of conveniently chosen subgroups of $W$. We hope to come back to this question in the future.

Other directions of investigation could involve the so-called Sutherland models, whose traditional study involves the same tools as described in this paper. However, it is expected that novel algebraic structures should be understood first. Indeed, already in the $A_{L}$ case, the symmetry algebra for spin Sutherland models is related to the Yangian of $\mathfrak{g l}_{N}$, a deformation of the half-loop algebra. For the $B_{L}$ case, the reflection algebra appears (when choosing the same spin representation as in Section 5.1) which is a deformation of the twisted half-loop algebra of order 2. However, for higher order twisting, the corresponding deformations are not known.

Finally, the symmetry algebras of the different spin Calogero models obtained in this paper might be useful to compute their eigenstates. Indeed, in the case of the $A_{L}$ Sutherland model, the Yangian symmetry was the cornerstone of the explicit construction of the eigenvectors $[11,12]$. It is based on representation theory of the Yangian and, in particular, on the construction of the Gelfand-Zetlin bases [29] which uses the knowledge of the maximal Abelian subalgebra containing the center. Let us remark that strangely enough, the situation in the case of the halfloop algebra seems more complicated. Indeed, the maximal Abelian subalgebra of the half-loop

algebra has been discovered 10 years after the one of the Yangian [30]. Thus, we hope that the present paper provides one additional motivation to look for the maximal Abelian subalgebra of the twisted cases. 


\section{Acknowledgements}

N.C. would like to thank the hospitality of the Centre for Mathematical Science, City University, where this work was initiated.

\section{References}

[1] Dunkl C.F., Differential-difference operators associated to reflection groups, Trans. Amer. Math. Soc. 311 (1989), 167-183.

[2] Calogero F., Solution of a three-body problem in one dimension, J. Math. Phys. 10 (1969), 2191-2196. Calogero F., Ground state of a one-dimensional N-body system, J. Math. Phys. 10 (1969), 2197-2200. Calogero F., Solution of the one-dimensional $N$-body problems with quadratic and/or inversely quadratic pair potentials, J. Math. Phys. 12 (1971), 419-436, Erratum, J. Math. Phys. 37 (1996), 3646.

[3] Sutherland B., Quantum many-body problem in one dimension: ground state, J. Math. Phys. 12 (1971), 246-250.

Sutherland B., Quantum many-body problem in one dimension: thermodynamics, J. Math. Phys. 12 (1971), 251-256.

Sutherland B., Exact results for a quantum many-body problem in one dimension, Phys. Rev. A 4 (1971), 2019-2021.

[4] Olshanetsky M.A., Perelomov A.M., Quantum integrable systems related to Lie algebras, Phys. Rep. 94 (1983), 313-404.

[5] Polychronakos A.P., Exchange operator formalism for integrable systems of particles, Phys. Rev. Lett. 69, 703-705.

[6] Minahan J.A., Polychronakos A.P., Integrable systems for particles with internal degrees of freedom, Phys. Lett. B 302 (1993), 265-270, hep-th/9206046.

[7] Hikami K., Wadati M., Integrability of Calogero-Moser spin system, J. Phys. Soc. Japan 62 (1993), 469-472.

[8] Bernard D., Gaudin M., Haldane F.D.M., Pasquier V., Yang-Baxter equation in long-range interacting systems, J. Phys. A: Math. Gen. 26 (1993), 5219-5236, hep-th/9301084.

[9] Faddeev L.D., Reshetikhin N.Y., Takhtajan L.A., Quantization of Lie groups and Lie algebras, Leningrad Math. J. 1 (1990), 193-225.

[10] Drinfel'd V.G., Hopf algebras and the quantum Yang-Baxter equation, Sov. Math. Dokl. 32 (1985), 254-258. Drinfel'd V.G., Quantum groups, in Proceedings of the International Congress of Mathematicians, Vols. 1,2 (Berkeley, Calif., 1986), Amer. Math. Soc., Providence, RI, 1987, 798-820.

[11] Takemura K., Uglov D., The orthogonal eigenbasis and norms of eigenvectors in the spin CalogeroSutherland model, J. Phys. A: Math. Gen. 30 (1997), 3685-3717, solv-int/9611006.

[12] Takemura K., The Yangian symmetry in the spin Calogero model and its applications, J. Phys. A: Math. Gen. 30 (1997), 6185-6204, solv-int/9701015.

[13] Uglov D., Yangian Gelfand-Zetlin bases, $\mathfrak{g} l_{N^{-}}$Jack polynomials and computation of dynamical correlation functions in the spin Calogero-Sutherland model, Comm. Math. Phys. 191 (1998), 663-696, hep-th/9702020.

[14] Ha Z.N.C., Haldane F.D.M., On models with inverse-square exchange, Phys. Rev. B 46 (1992), 9359-9368, cond-mat/9204017.

[15] Haldane F.D.M., Ha Z.N.C., Talstra J.C., Bernard D., Pasquier V., Yangian symmetry of integrable quantum chains with long-range interactions and a new description of states in conformal field theory, Phys. Rev. Lett. 69 (1992), 2021-2025.

[16] Bernard D., Pasquier V., Serban D., A one dimensional ideal gas of spinons, or some exact results on the XXX spin chain with long range interaction, hep-th/9311013.

[17] Hikami K., Yangian symmetry and Virasoro character in a lattice spin system with long-range interactions, Nuclear Phys. B 441 (1995), 530-548.

[18] Murakami S., Wadati M., Connection between Yangian symmetry and the quantum inverse scattering method, J. Phys. A: Math. Gen. 29 (1996), 7903-7915.

[19] Mintchev M., Ragoucy E., Sorba P., Zaugg Ph., Yangian symmetry in the nonlinear Schrödinger hierarchy, J. Phys. A: Math. Gen. 32 (1999), 5885-5900, hep-th/9905105. 
[20] Uglov D.B., Korepin V.E., The Yangian symmetry of the Hubbard model, Phys. Lett. A 190 (1994), 238-242, hep-th/9310158.

[21] Sklyanin E.K., Boundary conditions for integrable quantum systems, J. Phys. A: Math. Gen. 21 (1988), $2375-2389$.

[22] Caudrelier V., Crampé N., Integrable $N$-particle Hamiltonians with Yangian or reflection algebra symmetry, J. Phys. A: Math. Gen. 7 (2004), 6285-6298, math-ph/0310028.

[23] Humphreys J.H., Reflection groups and Coxeter groups, Cambridge Studies in Advanced Mathematics, Vol. 29, Cambridge University Press, Cambridge, 1990.

[24] Belavin A.A., Drinfel'd V.G., Solutions of the classical Yang-Baxter equation for simple Lie algebras, Funct. Anal. Appl. 16 (1982), 159-180.

Belavin A.A., Drinfel'd V.G., Classical Yang-Baxter equation for simple Lie algebras, Funct. Anal. Appl. 17 (1983), 220-221.

Belavin A.A., Drinfel'd V.G., Triangle equation and simple Lie algebras, Soviet Sci. Rev. Sect. C Math. Phys. Rev., Mathematical Physics Reviews, Vol. 4, Harwood Academic Publ., Chur, 1984, 93-165.

[25] Crampé N., Young C.A.S., Integrable models from twisted half-loop algebras, J. Phys. A: Math. Theor. 40 (2007), 5491-5509, math-ph/0609057.

[26] Yamamoto T., Multicomponent Calogero model of $B_{N}$-type confined in a harmonic potential, Phys. Lett. A 208 (1995), 293-302, cond-mat/9508012.

[27] Inozemtsev V.I., Sasaki R., Universal Lax pairs for spin Calogero-Moser models and spin exchange models, J. Phys. A: Math. Gen. 34 (2001), 7621-7632, hep-th/0106164.

[28] Quesne C., An exactly solvable three-particle problem with three-body interaction, Phys. Rev. A 55 (1997), 3931-3934, hep-th/9612173.

[29] Nazarov M., Tarasov V., Yangians and Gelfand-Zetlin bases, Publ. Res. Inst. Math. Sci. 30 (1994), 459-478, hep-th/9302102.

[30] Talalaev D., Quantization of the Gaudin system, hep-th/0404153. 